\title{
ERRATUM
}

\section{Construction of maximally localized Wannier functions}

\author{
Junbo Zhu (筑俊博 $)^{1}$, Zhu Chen $(\text { 陈竹 })^{2}$, Biao Wu (吴飙 $)^{1,3,4,5, \dagger}$ \\ ${ }^{1}$ International Center for Quantum Materials, School of Physics, Peking University, Beijing 100871, China \\ ${ }^{2}$ Institute of Applied Physics and Computational Mathematics, Beijing 100088, China \\ ${ }^{3}$ Collaborative Innovation Center of Quantum Matter, Beijing 100871, China \\ ${ }^{4}$ Wilczek Quantum Center, College of Science, Zhejiang University of Technology, Hangzhou 310014, China \\ ${ }^{5}$ Synergetic Innovation Center for Quantum Effects and Applications (SICQEA), \\ Hunan Normal University, Changsha 410081, China \\ Corresponding author.E-mail: ${ }^{\dagger}$ wubiao@pku.edu.cn \\ Received January 3, 2017; accepted January 6, 2017
}

Erratum to: Front. Phys. 12(5), 127102 (2017)

DOI: $10.1007 / \mathrm{s} 11467-016-0628-8$

In the original publication of the article, the funding information in the Acknowledgements was incomplete. A complete form is provided in this erratum as follows: This work was supported by the National Basic Research Program of China (Grants Nos. 2013CB921903 and 2012CB921300) and the National Natural Science Foundation of China (Grants Nos. 11274024, 11334001, 11429402, and 11404026).

The online version of the original article can be found at http://dx.doi.org/10.1007/s11467-016-0628-8 